AperTO - Archivio Istituzionale Open Access dell'Università di Torino

\title{
Intravitreal anti-VEGF agents and cardiovascular risk
}

\section{This is the author's manuscript}

Original Citation:

Availability:

This version is available http://hdl.handle.net/2318/1723677

since 2020-01-16T19:10:50Z

Published version:

DOI:10.1007/s11739-019-02253-7

Terms of use:

Open Access

Anyone can freely access the full text of works made available as "Open Access". Works made available under a Creative Commons license can be used according to the terms and conditions of said license. Use of all other works requires consent of the right holder (author or publisher) if not exempted from copyright protection by the applicable law. 
Massimo Porta, Elio Striglia

\section{INTRA-VITREAL ANTI-VEGF AGENTS AND CARDIOVASCULAR RISK}

Department of Medical Sciences

University of Turin

Corso AM Dogliotti 14

10126 Torino, Italy

Corresponding author:

Prof. Massimo Porta, MD PhD

massimo.porta@unito.it

Tel. +390116705214

ResearcherID: G-6146-2011

ORCID: 0000-0002-3407-6017 


\begin{abstract}
Antagonists of Vascular Endothelial Growth Factor (Anti-VEGF) are widely administered by intravitreal injection for the treatment of ocular pathologies such as Age-related Macular Degeneration, Diabetic Macular Edema, Proliferative Diabetic Retinopathy and occlusion of retinal vessels. Anti-VEGF agents, in particular bevacizumab, were introduced in oncology to inhibit tumor-induced angiogenesis feeding neoplastic tissues. Subsequently, other specific agents were developed for intraocular administration. Whereas systemic administration of anti-VEGF agents in oncology is burdened by increased risk of arterial hypertension and embolism, agents administered for ophthalmic indications are delivered locally into the eye globe in much smaller quantities. Nevertheless, clinical observations have raised the possibility that, even in these conditions, anti-VEGF agents may increase cardiovascular risk in patients who, being elderly and/or diabetic, are intrinsically prone to such events. This paper aims at reviewing the current knowledge on VEGF and its pharmacologic antagonists from mechanistic and side-effect points of view, with specific reference to patients with sight-threatening conditions. Internists should be aware of the need to collaborate with ophthalmologists and pharmacovigilance operators to define as best as possible the risk/benefit balance of intravitreal agents in patients who might lose their sight if left untreated, or increase their risk of suffering a cardiovascular event if treated.
\end{abstract}

\title{
Key Words:
}

Vascular Endothelial Growth Factor, diabetes mellitus, diabetic retinopathy, anti-VEGF agents, cardiovascular risk.

\section{Glossary:}

AMD = Age-related Macular Degeneration

$\mathrm{BRB}=$ Blood Retinal Barrier

CSME = Clinically Significant Macular Edema

$\mathrm{DME}=$ Diabetic Macular Edema

DR = Diabetic Retinopathy

HIF- $1 \alpha=$ Hypoxia Inducible Factor $1 \alpha$

PDR = Proliferative Diabetic Retinopathy

RVO $=$ Retinal Vessel Occlusion

VEGF $=$ Vascular Endothelial Growth Factor 


\section{Introduction}

Antagonists of Vascular Endothelial Growth Factor (Anti-VEGF) are widely administered by intravitreal injection for the treatment of ocular pathologies such as Age-related Macular Degeneration (AMD), Diabetic Macular Edema (DME), Proliferative Diabetic Retinopathy (PDR) and Retinal Vessel Occlusion (RVO). Apart from economic and regulatory issues which will not be discussed in this review, their administration presents a number of potential clinical problems because they may intrinsically increase cardio-embolic risk in patients who, being elderly and/or diabetic, are already prone to such events. Consequently, internists should be aware of the need to collaborate with ophthalmologists and pharmacologists or pharmacovigilance operators to define as best as possible the risk/benefit balance of intravitreal agents in patients who might lose their sight if left untreated, or increase their risk of suffering a heart attack or stroke if treated.

This paper aims at reviewing the current knowledge on VEGF and its pharmacologic antagonists from mechanistic and side effect points of view, with specific reference to patients with sight-threatening conditions.

\section{VEGF and angiogenesis}

VEGF plays a key role in the process of angiogenesis, both in physiological conditions, such as wound repair and endometrial reconstruction during the menstrual cycle, and in tumor growth or retinal neovascularization during PDR, retinopathy of prematurity, and sequelae of retinal vein thrombosis. Senger et al (1) described in 1983 a "Vascular Permeability Factor" (VPF) with the ability to increase vascular permeability. Ferrara and Henzel, in 1989, highlighted its mitotic effect on endothelial cells and proposed the name VEGF (2). Later, it was understood that there is a whole family of VEGFs: VEGF-A (the most important, usually referred to), VEGF-B, -C, and -D (implicated in embryonic and lymphatic angiogenesis), and placental PIGF, which may act to promote uterine angiogenesis and vascular permeability within the placental bed (3).

VEGF isoforms are the result of different splicing of the same 8-exon gene. The first product of transcription leads to forms that are structurally similar but have specific functions. Further molecules of the same family are: VEGF-E, of viral origin, and VEGF-F isolated from snake venom (4-5). VEGFs are produced, among others, by endothelial cells, pericytes, keratinocytes, fibroblasts, macrophages and placenta. They bind to trans-membrane tyrosine kinase receptors, inducing their dimerization and trans-phosphorylation. These receptors have an extracellular domain similar to immunoglobulins, a portion located in the thickness of the cell membrane and an intra-cytoplasmic one. The VEGFR-2 receptor (KDR/FLK1) mediates almost all the effects of VEGF-A, while VEGFR-1 (FIt-1) appears to modulate the activity of VEGFR-2 (6). A third receptor (VEGFR-3) binds VEGF-C and D. Finally, it was recently found that Neuropilin-1, a trans-membrane protein lacking tyrosine kinase activity, acts as a co-receptor for VEGF-A (7) (Figure 1) 
There are two isoforms of VEGF-A, coded 165-a and 165-b. The former increases vascular permeability, promotes the development of new vessels and has a pro-inflammatory effect, whereas VEGF 165-b has opposite properties as it inhibits new vascularization and is an anti-inflammatory, cyto- and neuroprotective factor (8). VEGF 165-a is increased, relative to 165-b, in PDR (9) and appears to promote inflammation and retinal exudates. Indeed, the development of new vessels from pre-existing vessels through morphogenesis requires endothelial cell destabilization, increased vascular permeability, mesenchymal transition of endothelial cells, proliferation and differentiation of new endothelial cells (10). Such destabilization requires the intervention of angiopoietin 2, which is produced by the same cells and induces the transition to mesenchymal cells expressing markers such as calponin, snail, and transgeline FSP1 (11). These cells detach from the others and lose their relationship with supporting pericytes, becoming at high risk of "anoikis", a type of apoptosis due to the lack of adhesive consent with the other cells and the basal membrane. However, they are "saved" by VEGF, which activates the anti-apoptotic PI3K-AKT pathway and causes them to proliferate and migrate to form new immature vessels (angiogenic switch and sprouting) (12).

\section{Role of VEGF in systemic retinopathies}

Most retinal conditions associated with systemic diseases such as diabetes, hypertension and thrombosis are characterized by neovascularization consequent to vascular occlusion and ischemia.

Hypoxia stimulates the release of Hypoxia Inducible Factor $1 \alpha$ (HIF-1 $1 \alpha)$, which is usually hydroxylated in its proline residues by prolyl-hydroxylases (a reaction that requires oxygen) and degraded by ubiquitin VHL in the proteasome within a "futile cycle" (13-14). In hypoxia, HIF-1 $\alpha$ is not adequately hydroxylated and, consequently, not degraded and enters the nucleus where it binds to HIF-1 $\beta$. This complex stimulates the transcription of the VEGF-A gene (15) by endothelium, pericytes and retinal pigment epithelium (13-14). The free radical nitric oxide (NO) produced by endothelial NO Synthase (eNOS) plays the role of mediator in the angiogenic response supported by VEGF either through the activation of PI3K or through the PLCgamma pathway. Once activated, both pathways converge on AKT phosphorylation of eNOS, releasing NO. VEGF also regulates vascular permeability by promoting endocytosis of VE-Cadherin (also known as cadherin 5) and regulation of Rho GTPase at the cell surface (16).

Thus, NO is crucial for the regulation of cytoskeletal architecture, leading to reversible changes in vascular permeability in vivo. However, the molecular mechanisms through which endogenous NO modulates endothelial permeability are not completely clear (17). Hence, VEGF is necessary for the survival of retinal endothelial cells too. Experimental studies in animals show that local inhibition of VEGF causes thinning and atrophy of the choriocapillaris and degeneration of photoreceptors (18).

There are many other regulatory factors of retinal angiogenesis, acting in response to hypoxic stimuli (1920). Some are inhibitors, such as thrombospondin for fibroblasts, which cause proliferating cells to release 
Fas ligand in a soluble form. This factor has no action on endothelial cells of mature vessels but induces apoptosis of immature ones in new vessels, which express a specific receptor (21). Null mice that do not produce thrombospondin exhibit marked vascularization and tumor growth (22). Pigment Epithelium Derived Factor (PEDF) is important for the integrity of the Blood-Retinal Barrier (BRB) and its deficiency seems to favor proliferation of retinal vessels and the development of DME (23). Basic fibroblast growth factor (bFGF) can also induce angiogenesis. Systemic administration of nitro-L-arginine methyl ester (LNAME), a NO synthase inhibitor, to rabbits bearing a corneal implant blocked VEGF but not bFGF induced angiogenesis: bFGF induce angiogenesis by different mechanistic pathway (24).

The imbalance between these factors promotes the transition from non-proliferative to proliferative retinopathy. Loss of autoregulation and capillary occlusions, especially in the peripheral retina, are the initial events providing the hypoxic stimulus that increases release of HIF-1 $\alpha$ (25). At the same time, factors closely related to hyperglycemia, such as AGE, PKC, RAS, IGF-1, FGF, and PDGF, contribute to stimulate the release of VEGF-A (26-27).

New vessels originate from small and medium caliber venules, though not capillaries. However, the first visible lesions, microaneurysms developing at the edge of non-perfused retinal areas, involve retinal capillaries. Microaneurysms are regarded as unsuccessful attempts to give rise to new-vessels, and their count in early diabetic retinopathy (DR) is an important prognostic indicator for its worsening (28). New vessels represent an attempt to vascularize ischemic areas in the peripheral retina. Unfortunately, the consequences are harmful because new vessels bleed easily and are associated to fibrosis due to myofibroblasts partly derived from circulating fibrocytes and partly from the same endothelial cells undergoing the above-mentioned mesenchymal transition (Figure 2) (29). In 1994, Aiello et al measured VEGF in intra-ocular fluids of 164 patients with retinal neovascularization associated with diabetic retinopathy, central retinal vein occlusion and rubeosis iridis, who had been subjected to vitrectomy or cataract surgery. They found increased levels of VEGF in those with proliferative compared to those with non-proliferative DR, quiescent PDR or who were not diabetic and were able to observe reduction of VEGF after laser treatment, confirming the efficacy of pan-retinal photocoagulation in reducing hypoxia by photodynamic ablation of the ischemic peripheral retina. In addition, VEGF stimulated the growth of retinal endothelial cells in vitro, as did vitreous fluid containing measurable VEGF, and this stimulation was inhibited by VEGF-neutralizing antibodies (30).

The other type of high-risk DR, DME, is today the leading cause of visual impairment in diabetes. Initially citotoxic, from intracellular accumulation of sorbitol, lactate and phosphates, DME becomes vasogenic, due to the intervention of inflammatory factors that loosen the tight junctions of the BRB. Small cystic fluid collections form in the inner layer of the macular area and can extend over the retinal surface with detachment of the neuro-retina (31). Both DME and PDR are mediated by an increase in VEGF-A 165a (32). 
Intravitreal administration of VEGF-A 165 b reduces retinal and choroidal vascularization (33-34) and increases the release of protective factors (35).

\section{Anti-VEGF agents and cardiovascular risk}

Anti-VEGF agents, in particular Bevacizumab, were initially introduced in oncology to inhibit tumor-induced angiogenesis feeding neoplastic tissues. Subsequently, Bevacizumab and other specific agents were developed for intraocular administration in the treatment of AMD, high-risk DR and other conditions characterized by retinal edema and/or neovascularization (36-37). Since VEGF protects blood vessel integrity, prolonged systemic administration of anti-VEGF agents in oncology is burdened by side effects (due to the lack of normal function of VEGF) as increased risk of arterial hypertension and embolism (Figure 3) (38-39). Hypertension is commonly observed during bevacizumab systemic treatment for cancer. All these patients are recommended to have blood pressure monitored every two to three weeks and in case of hypertension to initiate anti-hypertensive therapy. Hypotheses for the mechanisms of hypertension induced by systemically administered Bevacizumab include: increased vascular tone due to inhibition of VEGF-mediated vasodilatation with decreased NO and prostacyclin, increased endothelin-1, reactive oxygen species (ROS) and arterial stiffness, reduction of capillary density, activating of renin-angiotensin system with renal injury (40).

When used for ophthalmic indications, these agents are administered locally into the eye globe and in much smaller quantities but many studies have investigated the possibility that anti-VEGF agents may increase cardiovascular events in patients with diabetes, old age or both even if administered intraocularly. Some considerations should be made in this respect:

- Diabetes per se is associated with a risk of myocardial infarction equivalent to that of non-diabetic people who have already suffered a heart attack (41), so much so that the American Diabetes Association and American Heart Association recommend to consider diabetes a coronary artery heart disease equivalent rather than a mere risk factor (42).

- DR is a manifestation of generalized micro-and macrovascular degeneration and is associated with a further increase in cardiovascular risk (43-44).

- Type 2 diabetic patients with retinopathy are at greater risk of small vessel occlusions in the brain, indicating that the central nervous system may be as much a site of microangiopathy as the retina and kidneys (45).

- The hospitalization rate for stroke and myocardial infarction in individuals with DME is respectively 2 and 2.5 times higher than in diabetic patients without DME (46).

- Retinal lesions, even isolated small microhemorrhages and cotton wool spots, are found in up to $10 \%$ of the non-diabetic, non-hypertensive population and are associated with increased risk of ischemic heart disease, stroke and heart failure (47). 
Anti-VEGF agents used in ophthalmology include Bevacizumab (Avastin), Pegaptanib (Macugen), Ranibizumab (Lucentis) and Aflibercept (Eylea) (Fig 4). Bevacizumab is a humanized function-blocking monoclonal murine antibody with a molecular weight of $149 \mathrm{kDa}$. It was the first anti-VEGF agent approved by the FDA for systemic use in oncology. Pegaptamib (no longer in use) is a pegylated aptamer, a small RNA fragment with a molecular weight of $5 \mathrm{kDa}$ and is the only anti-VEGF designed to bind only the VEGF 165 isoform blocking its interaction with its receptors on endothelial cells. Ranibizumab is a recombinant humanized immunoglobulin G1k isotype antibody fragment with a molecular weight of $48 \mathrm{kDa}$. It can bind all VEGF-A isoforms. Ranibizumab is three times smaller than bevacizumab, and its intravitreal half-life is $75 \%$ shorter. Aflibercept, the latest approved in Europe and America, is a $115 \mathrm{kDa}$ soluble decoy receptor is made up by the second domain of VEGF-R1 and the third domain of VEGF-R2, fused to the Fc region of a human IgG1. It acts as a competitive inhibitor of VEGF, also binding to PIGF.

Two more drugs will become available in the near future: Combercept and Abicipar Pegol. The former, currently approved in China, is a $141 \mathrm{kDa}$ recombinant fusion protein of the VEGF receptor domains with the Fc fragment of a human immunoglobulin. The difference between Combercept and Aflibercept is the presence of a fourth VEGF-R2 binding domain in Combercept, which stabilizes the receptor-ligand complex (48). Abicipar Pegol, already used in oncology and asthma, binds with high affinity to all soluble isoforms of VEGF-A, similar to Ranibizumab, but has smaller molecular weight (34 kDa vs 48 kDa), longer ocular half-life and higher efficacy, as 6-8 injections are comparable to 13 Ranibizumab injections (49).

Bevacizumab does not have an ophthalmic indication and is administered off label in doses on average 150 times lower than those used in oncology (50), whereas the other three are registered specifically for ophthalmic use.

Bevacizumab and Aflibercept maintain plasma levels close to their IC50 (the concentration necessary to inhibit $50 \%$ of VEGF biological activity) as long as 30 days after intravitreal administration, which makes cardiovascular side-effects potentially possible (51). Ranibizumab has a lower half-life because, in contrast with the other two molecules, does not contain the Fc component (52). Matsuyama et al showed that the blood concentrations of both VEGF and PDGF are reduced within 24 hours of a single intravitreal administration of Bevacizumab and that the effect lasts for at least 1 month (53). Avery et al in 2017 demonstrated that Ranibizumab clears from the bloodstream more quickly than Bevacizumab or Aflibercept. Aflibercept results in the greatest reduction in plasma free VEGF, whereas Ranibizumab produces the smallest decrease in plasma free VEGF. Generally, the reduction in plasma free VEGF levels correlates with elevated levels of circulating anti-VEGF (54).

Lee et al in 2018 assessed VEGF plasma levels after treatment with intravitreous Aflibercept, Bevacizumab or Ranibizumab in 436 participants at baseline and 4, 52 and 104 weeks later. Their study suggests that 
decreases in plasma VEGF levels are greater with Aflibercept or Bevacizumab compared to Ranibizumab confirming Avery's findings (55).

Usually, these agents are injected once a month for 3 months and can be further administered monthly or every 2 months, according to clinical judgement based upon anatomical and visual outcomes. In 2019 Busch et al, reported the 2-year follow-up outcomes of non responding DME after three monthly anti-VEGF injections vs early switch to dexamethasone (DEX) implant and showed a beneficial effect of an early switch to DEX implant in DME non responders, at month 12, maintained during the second year, suggesting a role for VEGF-independent inflammatory mechanisms. A later switch from anti-VEGF to steroids still provides a significant improvement of visual acuity (56) .

Kamba et al reported a decrease in both NO and prostacyclin after systemic anti-VEGF administration, accompanied by increased arterial pressure and endothelial cell detachment, potentially favoring the occurrence of thrombotic events (38). Zarbin, in 2018, recognizing that systemic administration of antiVEGF agents is associated with these complications, stated that the reduction in free plasma VEGF levels following intravitreal injection may lead to similar consequences. Although the frequency of systemic serious adverse events after intravitreal administration is low, patients with recent stroke or other events may be at higher risk of systemic complications (57). More so, the possibility that repeated intraocular administration of anti-VEGF agents is associated with systemic disorders is real, although it remains difficult to discriminate how much of the risk is due to the drug and how much to the underlying systemic pathology itself, old age and lifestyle.

\section{Clinical trials, reviews and meta-analyses}

Several studies were carried out over the last 20 years, including systematic reviews and meta-analyses, to shed light on this problem but the results are still inconclusive. Potential biases include the facts that patients enrolled in clinical trials, and therefore in the meta-analyses that followed, are often not representative of people subjected to intravitreal treatments in real life.

The Pan American Collaborative Study group (PACORES), published in 2008, was a retrospective survey of 1173 patients with and without diabetes, followed monthly for one year after treatment with bevacizumab. Systemic adverse events were as follows: 7 (0.59\%) cases of acute elevation of systemic blood pressure, 6 $(0.5 \%)$ vascular accidents, $5(0.4 \%)$ myocardial infarctions, $2(0.17 \%)$ iliac artery aneurysms, $2(0.17 \%)$ toe amputations and $5(0.4 \%)$ deaths. Ocular complications included $7(0.16 \%)$ cases of bacterial endophthalmitis, $7(0.16 \%)$ tractional retinal detachments, $4(0.09 \%)$ cases of uveitis and 1 each of retinal detachment and vitreous detachment (58). Rajendram et al, in 2012, reported the 2-year outcomes of the BOLT study, a prospective randomized controlled trial evaluating intravitreous Bevacizumab in 80 patients with persistent clinically significant macular edema (CSME). This study provided evidence supporting longer-term use of intravitreous Bevacizumab for CSME but found myocardial infarction in $4.8 \%$ of patients 
and one fatality. However, no significant differences were noticed between the patients on Bevacizumab and those treated with laser, suggesting that off label Bevacizumab may be safe in patients with DME (59). On the other hand, one more recent retrospective case-control study, with a maximum follow-up of 73 months, enrolled over 5000 patients with AMD treated with Bevacizumab and showed an increased risk of death from all causes (OR 1.69; $\mathrm{Cl} 95 \%$ 1.54-1.84, p<0.001) (60).

RISE and RIDE, two phase-3, randomized, multicenter, double-masked trials enrolled 759 patients with DME. These were randomized equally to 0.3 or $0.5 \mathrm{mg}$ monthly Ranibizumab or sham injections. The incidence of adverse events was higher in the patients who received $0.5 \mathrm{mg}$ Ranibizumab compared to those receiving $0.3 \mathrm{mg}$ Ranibizumab or sham $(19.7,16.8$ and 13.1 respectively). The incidence of central nervous system hemorrhage, congestive heart failure, hypertension, gastrointestinal perforation, proteinuria and wound-healing complications appeared increased in patients on Ranibizumab (61). In 2014 the extension of RESTORE, a phase-3 randomized multicenter study, evaluated long-term efficacy and safety of Ranibizumab during 3 years of treatment in 240 patients with DME. No cases of endophthalmitis, retinal tear or retinal detachment were reported. The most frequently reported adverse effects over 3 years were cataract (16.3\%) and nasopharyngitis (23.3\%). Eight deaths were reported during the extension study, but none were suspected to be related to the drug (62).

Aflibercept was studied in the VIVID and VISTA trials, which compared efficacy and safety of intravitreal injection against laser photocoagulation for DME over 3 years (148 week) in 872 eyes, showing good safety (63). However, two years later (2018), Sobolewska et al, showed a potential risk of thromboembolic events for Aflibercept explained by increased activation of the fibrinogen receptor GPIIb/IIla and the presence of a direct interaction between the drug and platelets (64).

However, most trials are sponsored by pharmaceutical companies for registration purposes, adding potential biases. For example, in VIVID and VISTA adverse effects were not related to Aflibercept if they occurred 30 or more days after the last injection even if we know that, as mentioned above, the drug is still present in the blood. Finally, the interactions between anti-VEGF and other therapies and the remark that angiogenesis is partially independent of VEGF (65-66) make it difficult to analyze the results of each study. Some reviews comparing Bevacizumab to Ranibizumab suggested that the former increases systemic events by $20-35 \%$ (67-68). In particular, two randomized controlled trials in patients with AMD: CATT, including 128 patients in the USA, and IVAN on 610 patients in the UK suggest a marginal superiority of Ranibizumab in terms of visual acuity gain. In IVAN there were more atherothrombotic events and heart failure with Ranibizumab. Other adverse events were more frequent with Bevacizumab, including infections, palpitations, and gastrointestinal disorders. However, CATT and IVAN were not powered to look at safety, as their sample sizes were too small (69).

Other authors have associated Ranibizumab with increased risk of extraocular bleeding (70). However, publication bias should be considered, and some conclusions were not confirmed in 2014 by Moja et al, 
who also examined unpublished studies. Their systematic review of non-industry sponsored studies could not reveal differences between intravitreal Bevacizumab and Ranibizumab for death and all serious systemic adverse events in the first two years of treatment, with the exception of gastrointestinal disorders, for which there was a higher risk with Bevacizumab (71).

In a meta-analysis of 13 RCT, Cheng et al, in 2012, evaluated 4942 patients with AMD, DR and RVO treated with anti-VEGF vs controls without showing increased arterial thromboembolic events (72). In 2014, Thulliez et al, in a meta-analysis of 21 trials that evaluated 9557 patients with DME, AMD and RVO subjected to anti-VEGF therapy with Bevacizumab or Ranibizumab compared to no anti-VEGF treatment, concluded for no increase in the risk of major cardiovascular events (OR 1.18; CI 95\% 0.81-1.71), stroke (OR 1.61; Cl 95\% 0.85-3.05), overall mortality (OR 1.53; Cl 95\% 0.92-2.56), non ocular hemorrhagic events (OR 1.42; Cl 95\% 0.95-2.13), hypertension (OR 0.97; Cl 95\% 0.71-1.32), or myocardial infarction (OR 0.92; Cl 95\% 0.54-1.59). Furthermore, no significant difference was found between patients treated with lower or higher doses of Ranibizumab ( $0.3 \mathrm{vs} 0.5 \mathrm{mg}$, only the latter being registered in Europe) with regard to the risk of major cardiovascular events (OR 0.86; Cl 95\% 0.62-1.21) and extraocular hemorrhages (OR 0.92; $\mathrm{Cl} 95 \%$ 0.67-1.26). However, that paper showed a greater risk of venous thromboembolism associated with Bevacizumab compared to Ranibizumab (OR 3.45; Cl 95\% 1.25-9.54) and an increased risk of non-fatal extraocular hemorrhages associated with Ranibizumab compared to controls not on anti-VEGF agents in patients with AMD (OR 1.55; Cl 95\% 1.01-2.44) (73).

Braithwaite et al in 2014 issued a Cochrane review about macular edema secondary to RVO, comparing anti-VEGF to sham injections or no treatment and showed improved visual outcomes in the anti-VEGF group without safety concerns (74).

Avery and Gordon in 2016 reported increased risk of death from all causes (OR 2.98; Cl 95\% 1.44-6.14, $\mathrm{p}=0.04$ ), cardiovascular accidents (OR 2.33; $\mathrm{Cl} 95 \%$ 1.04-5.22, $\mathrm{p}=0.04$ ) and vascular death (OR 2.51; $\mathrm{Cl} 95 \%$ 1.08-5.82, $p=0.03$ ) in 1328 patients with DME treated with Aflibercept or Ranibizumab compared to laser or sham. It should be noted, though, that they considered patients treated monthly with high doses for 2 years while other studies (which did not produce similar results) had enrolled patients subjected to less intensive and shorter treatments. The main reason for increased risk of death in the study by Avery and Gordon was ischemic stroke (75).

Zarbin et al in 2017 evaluated cardio- and cerebrovascular risk in DME with 0.3 or $0.5 \mathrm{mg}$ Ranibizumab vs sham or laser in a pooled-analysis that considered 6 phase- 2 and -3 registrative trials for a total of 1867 patients (1186 on Ranibizumab). Unlike Avery and Gordon, they did not report increased risk of death from all causes or systemic vascular events associated with the active drug (76).

Thulliez et al in 2018 published another overview considering 21 meta-analyses about AMD, DME and RVO, paying particular attention to the selection criteria in order to eliminate possible confounding factors and suggested that anti-VEGF do not increase the risk of systemic adverse events, although caution is advisable 
in AMD patients who may be at higher risk of hemorrhagic events when receiving Ranibizumab. They also stated that the increased risk of cardiovascular death reported by Avery and Gordon might have been due to patient old age and/or repeated higher doses of the drug (77).

Starr, Dalvin, et al authored a population-based, retrospective cohort study including 2541 patients with $A M D, D M E$ and RVO which suggested no increased risk of stroke (either ischemic or hemorrhagic), MI or death in patients receiving intravitreal anti-VEGF compared with control populations (78-79). In a Cochrane analysis of 2018 on data relevant to diabetic patients with DME at the longest available follow-up ( 1 or 2 years), Virgili et al (80) found that Aflibercept, Ranibizumab and Bevacizumab do not differ in terms of serious systemic adverse events. They noted that more than 25 million injections have been delivered worldwide and that, by now, we should be able to draw some firm conclusion about this problem.

In 2019 Maloney et al, reported the risk of systemic adverse events associated with intravitreal Anti-VEGF therapy for DME in routine clinical practice compared with controls treated with macular laser photocoagulation or intravitreal corticosteroid in a cohort of 23348 patients. Anti-VEGF treatment was not associated with an increased hazard ratio of cerebrovascular disease (HR 0.96; $\mathrm{Cl} 95 \% 0.65-1.41 ; \mathrm{p}=0.83$ ), major bleeding (HR 1.23; Cl 95\% 0.76-1.99; $\mathrm{p}=0.41$ ), or myocardial infarction (HR 1.03; Cl955 0.73 $1.441 ; p=0.88$ ) when compared with macular laser for DME; however there was an increased risk of post treatment all-cause hospital admission (HR 1.17; Cl 95\% 1.05-1.30; $p=0.01$ ) (81). However, risk of bias still makes estimates not precise enough for low-frequency events like arterial thromboembolism or death. Most industry-sponsored studies became open-label after one year of follow up and one large independent study found no difference among the three drugs at 2 years.

A synopsis of published studies is shown in Table 1.

In conclusion, the risk of atherothrombotic events is low compared with their natural incidence in elderly patients, and acceptable if we consider the advantage on vision gain. However, caution is necessary in patients with recent thromboembolic events or myocardial infarction. It could be safer to use drugs with short systemic half-life that do not accumulate in the serum, like Ranibizumab or Pegaptamib or alternate drugs with longer plasma half-lives, which have the Fc fragment, with drugs with short plasma half-lives, without Fc fragment (82).

\section{Is there a role for the internist?}

Systemic use of anti-VEGF agents in oncology is associated with increased risk of arterial hypertension, cardiovascular events and arterial embolism. However, it is not established whether also their intravitreal administration increases such risks. Limits in the above reported studies make the interpretation of available data uncertain. Moreover, it would be important to increase pharmacosurveillance, in order to uncover complications arising from the administration of these drugs in real life. A decision to administer 
anti-VEGF drugs should be made on the basis of a case by case risk/benefit analysis, taking into account the patient's comorbidities, age and current therapies. Of the three anti-VEGF agents available for intra-ocular administration, with different structures and half-lives, Bevacizumab might be associated with greater risks but is by far less expensive (very important in developing Countries). Ranibizumab has a shorter half-life and may involve fewer risks, while Aflibercept may be effective in the rescue of more advanced cases of DME. The decision to treat requires the collaboration not only between ophthalmologist, diabetologist and internist, but also pharmacologist and pharmacovigilance operators, weighing the need to save sight (DR and AMD remain major causes of blindness (83)) and the apparently minimal additional possibility of further increasing the already high cardiovascular risk related to diabetes and/or old age (82). Clinicians should minimize the dose and frequency of injections without compromising results case by case rather than adopting rigid monthly protocols. Abicipar Pegol which can be administered every 3 months after the loading phase, could be an interesting option in the next future (82). It should be underlined anyway that this review cannot provide indications about the best preferred use of anti-VEGF agents from a strictly ophthalmological point of view.

Finally, one should bear in mind that DR meets all the criteria for screening: DR is an important public healthcare problem and screening is simple, safe, and effective treatments are available. Internists should not overlook the importance of screening to detect patients at risk for sight-threatening DR at a stage when they can be effectively treated by less invasive options (laser) (84-85). However, once a need for anti-VEGF arises, they may be requested to assess the overall cardiovascular risk of individual candidates before intravitreal treatment, much like anesthesiologists assess overall risk before elective surgery. This may sound bizarre but the continuing evolution of medical knowledge and therapies carries the emergence of novel tasks and responsibilities, which include timely and correct patient information on the different options available, as established by legal and deontological considerations.

\section{Compliance with Ethical Standards}

This paper does not directly involve studies in humans or animals.

\section{Conflicts of interest}

MP has received speaker honoraria from Allergan, Novartis and SIFI. 


\section{References}

1) Senger DR, Galli SJ, Dvorak AM et al (1983) Tumor cells secrete a vascular permeability factor that promotes accumulation of ascites fluid. Science 219:983-985.

2) Ferrara N, Henzel WJ (1989) Pituitary follicular cells secrete a novel heparin-binding growth factor specific for vascular endothelial cells. Biochem Biophys Res Commun 161:851-858.

3) Shore VH, Wang TH, Wang CL et al (1997) Vascular endothelial growth factor, placenta growth factor and their receptors in isolated human trophoblast. Placenta 18(8):657-665.

4) Wise LM, Inder MK, Real NC et al (2012) The vascular endothelial growth factor (VEGF)-E encoded by orf virus regulates keratinocyte proliferation and migration and promotes epidermal regeneration. Cell. Microbiol 14:1376-90.

5) Yamazaki Y, Matsunaga Y, Tokunaga Y et al (2009) Snake venom Vascular Endothelial Growth Factors (VEGF-Fs) exclusively vary their structures and functions among species. J.Biol Chem 10, 284:9885-91.

6) Holmes K, Roberts O, Angharad M et al (2007) Vascular endothelial growth factor receptor 2: structure, function, intracellular signaling and therapeutic inhibition. Cellular Signalling vol $19 \mathrm{n} 10$ : 2003-12.

7) Fantin A, Herzog B, Mahmoud M et al(2014) Neuropilin 1 (NRP-1) hypomorphism combined with defective VEGF-A binding reveals novel roles for NRP-1 in developmental and pathological angiogenesis. Development 141:556-562.

8) Beasley_Long N, Hua J, Jehle T et al (2013) VEGF-A 165b is an endogenous neuroprotective splice isoform of vascular endothelial growth factor A in vivo and in vitro. Am J Pathol 183:918-929.

9) Ved N, Hulse RP, Bestall SM et al (2017) Vascular endothelial factor-A 165b ameliorates outer retinal barrier and vascular dysfunction in diabetic retina. Clin Sci 131(12): 1225-1243.

10) Kalluri R and Weinberg RA (2009) The basics of epithelial-mesenchymal transition. The journal of Clinical Investigation 119(6):1420-28.

11) Frisch SM, Francis H (1994) Disruption of epithelial cell-matrix interactions induces apoptosis. The Journal of Cell Biology 124 (4):619-626.

12) Liotta LA, Kohn E (2004) Anoikis: cancer and homeless cell. Nature 430:973-4.

13) Adams AP, Shima DT, Yeo KT et al (1993) Synthesis and secretion of vascular permeability factor/vascular endothelial growth factor by human retinal pigmental cells. Biochem Biophys Res Commun 193:631-638.

14) Aiello LP, Ferrara N, King GL (1994) Hypoxic regulation and bioactivity of vascular endothelial growth factor: characterization in retinal microvascular pericytes and pigment epithelial cells. Invest Ophthalmol Vis Sci:35:1868. 
15) Aiello LP, Gardner TW, King GL et al (1998) Diabetic Retinopathy. Diabetes Care 21:143-156.

16) Lin Ml et al (2006) Vascular endothelial growth factor signalling to endothelial nitric oxide synthase: more than a FleeTing moment. Clin Res 29:99(7):666-8.

17) Di Lorenzo A, Lin MI, Murata T et al (2013) eNOS derived nitric oxide regulates endothelial barrier function through VE-cadherin and Rho GTPases. Journal of Cell Science 126:5541-5552.

18) Semeraro F, Morescalchi F, Duse S et al (2015) Pharmacokinetic and Oharmacodynamic Properties of Anti_VEGF Drugs After Intravitreal Injection. Curr Drug Metab 16(7):572-84.

19) Ranieri G and Gasparini G (2001) Angiogenesis and angiogenesis inhibitors: a new potential anticancer therapeutic strategy. Current Drugs Targets Immune, Endocrine \& Metabolic Disorders $1: 241-253$

20) Yancopoulos GD, Davis S, Gale NW et al (2000) Vascular specific growth factors and blood vessel formation. Nature 407:242-248.

21) Kazerounian S and Lawler J (2018) Integration of pro and anti angiogenic signals by endothelial cells. J Cell Commun Signal 12(1):171-179.

22) Lawler J, Miao WM, Duquette M et al (2001) Thrombospondin-1 gene expression affects survival and tumor spectrum of p53 deficient mice. Am J Pathol 159:1949-56.

23) Simò R and Hernandez C (2014) Neurodegeneration in diabetic eye: new insights and therapeutic perspectives. Trend Endocrinol Metab 25:23-33.

24) Ziche M, Morbidelli L, Choudhuri R et al (1997) Nitric oxide synthase lies downstream from vascular endothelial growth factor-induced but not basic fibroblast growth factor-induced angiogenesis. J Clin Invest 1:99(11): 2625-34.

25) De Vriese AS, Verbeuren TJ, Van de Voorde J et al (2000) Endothelial dysfunction in diabetes. Br J Pharmacol 130:963-74.

26) Brownlee M (2001) Biochemistry and molecular cell biology of diabetic complications. Nature 4141 : 813-20.

27) Koya D, King GL (1998) Protein kinase C activation and the development of diabetic complications. Diabetes 47(6): 859-66.

28) Sjølie AK, Klein R, Porta M et al (2011) Retinal microaneurisms count predicts progression and regression of diabetic retinopathy. Post hoc results from the DIRECT Programme. Diabet Med 28:345-351.

29) Abu El-Asar AM, De Hertogh G, Van den Eynde K et al (2015) Myofibroblasts in proliferative diabetic retinopathy can originate from infiltrating fibrocytes and through endothelial-to mesenchymal transition (EndoMIT). Exp Eye es 132:179-89.

30) Aiello LP, Avery RL, Arrigg PG et al (1994) Vascular endothelial growth factor in ocular fluid of patients with diabetic retinopathy and other retinal disorders. NEJM 331:1480-87. 
31) Romero-Aroca P, Baget-Bernaldiz M, Pareia-Rios A et al (2016) Diabetic macular edema. Pathophysiology: vasogenic versus inflammatory. J Diab Res 2156273 Epub 2. http://dx.doi.org/10.1155/2016/2156273.

32) Perrin RM, Konopatsakaya O, Qiu Y et al (2005) Diabetic retinopathy is associated with a switch in splicing from anti to pro-angiogenic isoform of vascular endothelial growth factor. Diabetologia 48: 2422-2427.

33) Konopatsakaya O, Churchill AJ, Harper SJ et al (2006) VEGF 165b an endogenous C terminal splice variant of VEGF inhibits retinal neovascularization in mice. Mol Vis 12:626-632.

34) Hua J, Spee C, Kase $S$ et al (2010) Recombinant human VEGF 165b inhibits experimental choroidal neovascularization. Invest Ophthalmol Vis Sci 51:4282-4288.

35) Magnussen AL, Rennel ES, Hua J et al (2010) VEGF-A 165b is cytoprotective and antiangiogenic in the retina. Invest Ophthalmol Vis Sci 51:4272-4281.

36) Schmidt-Erfurth U, Lang GE, Holz FG et al (2014) The RESTORE extension Study Group. Three-Year Outcomes of individualized Ranibizumab Treatment in Patients with Diabetic Macular Edema: The RESTORE Extension Study. Ophthalmology 121(5):1045-53.

37) Gross JC, Glassman AR (2015) Writing Committee for the Diabetic Retinopathy Clinical Research Network. Panretinal photocoagulation vs intravenous ranibizumab for proliferative diabetic retinopathy: a randomized clinical trial. JAMA 314:2137-46.

38) Kamba T, Mc Donald DM (2007) Mechanisms of adverse effects of anti-VEGF therapy for cancer. $\mathrm{Br}$ J Cancer 96:1788-98.

39) Roodhart JM, Lanenberg MH, Witteveen E, Voest EE (2008) The molecular basis of class side effects due to treatment with inhibitors of the VEGF/VEGFR pathway. Curr Clin Pharmacol 3(2):132-43.

40) Li M, Kroetz DL (2018) Bevacizumab-induced hypertension: Clinical presentation and molecular understanding. Pharmacol Ther 182:152-160.

41) Haffner SM, Lehto S, Ronnemea T et al (1998) Mortality from coronary heart disease in subjects with type 2 diabetes and non diabetic subjects with and without prior myocardial infarction. NEJM 339:229-234.

42) Buse JB, Ginsberg HN, Bakris GL et al (2007) American Heart Association; American Diabetes Association:Primary prevention of cardiovascular diseases in people with diabetes mellitus: a scientific statement from the American Heart Association and the American Diabetes Association. Diabetes Care 30:162-172.

43) Zhu XR, Zhang YP, Bai L et al (2017) Prediction of risk of diabetic retinopathy for all-cause mortality, stroke and heart failure. Evidence from epidemiological, observational studies. Medicine 96: 3(e5894). doi: 10.1097/MD.0000000000005894 
44) Shoeibi N, Bonakdaran S (2017) Is There any Correlation between Diabetic Retinopathy and Risk of Cardiovascular Disease? Curr Diabetes Rev.13(1):81-86.

45) Sanahuja J, Alonso N, Diez J et al (2016) Increased burden of cerebral small vessel disease in patients with type 2 diabetes and retinopathy. Diabetes Care 39:1614-20.

46) Nguyen-Khoa BA, Goehring EL, Werther W et al (2012) Hospedalized cardiovascular events in patients with diabetic macular edema. BMC Ophthalmol 12: 11. doi: 10.1186/1471-2415-12-11.

47) Liew G, Wong TI, Mitchell P et al (2009) Retinopathy predicts coronary heart disease mortality. Heart 95:391-394.

48) Li X, Xu G, Wang Y et al (2014) Safety and efficacy of combercept in neovascular age-related macular degeneration:results from 12 month randomized phase 2 study: AURORA study. Ophthalmology 121(9):1740-47.

49) Pluckthun A (2015) Designed ankyrin repeat proteins (DARPns): binding proteins for research, diagnostics, and therapy. Annu Rev Phaarmacol Toxicol 55:489-511.

50) Michels S (2006) Is intravitreal bevacizumab (Avastin) safe? Br J Ophthalmol 90(11):1333-4.

51) Avery RL, Castellarin AA, Steinle NC et al (2017) Systemic pharmacokinetics and pharmacodynamics of intravitreal aflibercept, bevacizumab and ranibizumab. Retina 27: 1847-1858.

52) Xu L, Lu T, Tuomi L et al (2013) Pharmacokinetics of ranibizumab in patients with neovascular agerelated macular degeneration: a population approach. Invest Ophthalmol Vis Sci 54:1616-1624.

53) Matsuyama K, Ogata N, Matsuoka M et al (2010) Plasma levels of vascular endothelial growth factor and pigment epithelium-derived factor before and after injection of bevacizumab. $\mathrm{Br} \mathrm{J}$ Ophthalmol 94(9):1215-1218.

54) Avery RL et al (2017) Systemic pharmacokinetics and pharmacodynamics of intravitreal aflibercept, bevacizumab and ranibizumab. Retina 37(10):1847-58.

55) Lee et al (2018) Plasma vascular endothelial growth factor concentrations after intravitreous antivascular endothelial growth factor therapy for diabetic macular edema. Ophthalmology 125:10541063.

56) Busch C, Frazer-Bell S, Iglicki M et al (2019) Real-world outcomes of non-responding diabetic macular edema treated with continued anti-VEGF therapy versus early switch to dexamethasone implant:2-year results. Acta Diabetologica https:// doi.org/10.1007/s00592-019-01416-4.

57) Zarbin MA (2018) Anti-VEGF Agents and the Risk of Arteriothrombotic Events. Asia Pac J Ophthalmol 7(1):63-67.

58) Wu L, Martinez-Castellanos MA, Quiroz-Mercado et al (2008) Twelve month safety of intravitreal injections of bevacizumab (Avastin): results of the Pan American Collaborative Retina Study Group (PACORES). Graefes Arch Clin Exp Ophthalmol 246(1):81-87. 
59) Rajendram R, Fraser-Bell S, Kaines A et al(2012) A 2-year prospective randomized controlled trial of intravitreal bevacizumab or laser therapy (BOLT) in the mnagment of diabetic macular edema: 24 month data:report 3. Arch Ophthalmol 130(8): 972-9.

60) Hanhart J, Comaneshter DS, Freier Dror Y et al (2017) Mortality in patients treated with intravitreal bevacizumab for age related macular degeneration. BMJ Ophthalmol 17(1):189. doi: $10.1186 / \mathrm{s} 12886-017-0586-0$

61) Brown DM, Nguyen QD, Marcus DM et al (2013) Long-term outcomes of ranibizumab therapy for diabetic macular edema: the 36-month results from two phase III trials: RISE and RIDE. Ophthalmology 120(10):2013-2022.

62) Schmidt-Erfurth U, Lang GE, Holz FG et al (2014) Three-year outcomes of individualized ranibizumab treatment in patients with diabetic macular edema: the RESTORE extension study. Ophthalmology 121(5): 1045-53.

63) Hejer JS, Korobelnik JF, Brown DM et al (2016) Intravitreal aflibercept for diabetic macular edema: 148 week results from VISTA and VIVID studies. Ophthalmology 123:2376-2385.

64) Sobolewska B, Golenko J, Poeschel S et al (2018) Influence of aflibercept on platelet activation profile. Exp Eye Res 175:166-172.

65) Etulain J, Negrotto S, Tribulatti MV et al (2014) Control of angiogenesis by galectin involves the release of platelet derived proangiogenic factors. Plos One 9(4): e96402. doi: 10.1371/journal.pone.0096402.

66) Ameri H, Liu H, liu R et al (2014) TWEAK/Fn14 pathway is a novel mediator of retinal neovascularization. Invest Ophthalmol Cis Sci 1055(2):801-13.

67) Solomon SD, Lindsley K, VedulaSS et al (2014) Anti-vascular endothelial growth factor for neovascular age related macular degeneration. Cochrane Database Syst rev 8; cd005555244. doi: 10.1002/14651858.CD005139.pub4.

68) Wang WJ, Chen J, Zhang XL et al (2015) Bevacizumab versus ranibizumab for neovascular age related macular degeneration: a meta-analysis. Int J Ophthalmol 8(1):138-147.

69) Ahfat FG and Zaidi FH (2013) Bevacizumab vs ranibizumab - an appraisal of the evidence from CATT and IVAN. Eye (Lond):289-90.

70) Ueta T, Noda Y, Toyama T et al (2014) Systemic safety of bevacizumab versus ranibizumab for neovascular age related macular degeneration: systematic review and meta-analysis of randomized trials. Ophthalmology 121 (11):2193-2203.

71) Moja L, Lucenteforte E, Kwang KH et al (2014) Systematic safety of bevacizumab versus ranibizumab for neovascular age-related macular degeneration. Cochrane Database Sist Rev (9):cd 011230. doi: 10.1002/14651858.CD011230.pub2. 
72) Cheng JW, Cheng SW, Lu GC et al (2012) Effect of intravitreal anti-vascular endothelial growth factor therapy on the risk of arterial thromboembolic events: a meta-analysis. PloS One 7(7):e41325.

73) Thulliez M, Angoulevant D, De Lez ML et al (2014) Cardiovascular events and bleeding risk associated with intravitreal anti-vascular endothelial growth factor monoclonal antibodies: systematic review and meta-analysis. JAMA Ophthalmol 132(11):1317-1326.

74) Braithwaite T, Nanji AA, Lindsley K et al (2014) Anti-vascular endothelial growth factor for macular oedema secondary to central retinal vein occlusion. Cochrane Data Base Syst Rev (5) CD007325.

75) Avery RL, Gordon GM (2016) Systemic safety of prolonged monthly antivascular endothelial growth factor therapy for diabetic macular edema: a systematic review and meta-analysis. JAMA Ophthalmol 134(1):21-29.

76) Zarbin MA, Dunger-Baldauf C, Haskova Z et al (2017) Vascular safety of ranibizumab in patients with diabetic macular edema: a pooled analysis of patient-level data from randomized trials. JAMA Ophthalmol 135(5):424-431.

77) Thulliez M, Angoulevant D, Pisella PJ et al (2018) Overview of Systematic Reviews and Metaanalysis on Systemic Adverse events Associated with intravitreal Anti Vascular Endothelial Growth Factor Medication Use. JAMA Ophthalmol doi-101001. Published online March 22. doi: 10.1001/jamaophthalmol.2018.0002.

78) Starr MR, Dalvin LA, AbouChehade JE et al (2019) Classification of strokes in patients receiving intravitreal anti-vascular endothelial growth factor. Ophthalmic Surg Lasers Imaging Retina 50(5):2140-e157.

79) Dalvin LA, Starr MR, AbouChehade JE et al (2019) Association of Intravitreal Anti-Vascular Endothelial Growth Factor Therapy With Risk of Stroke, Miocardial Infarction and Death in Patients With Exudative Age-Related Macular Degeneration. JAMA Ophthalmol 137(5):483-490.

80) Virgili G, Parravano M, Evans JR et al (2018) Anti-vascular endothelial growth factor for diabetic macular edema: a network meta-analysis. Cochrane Database Syst Rev 2018 Oct 16; 10:CD007419.doi:10.1002/14651858,CD007419.pub6.

81) Maloney MH, Schilz SR, Herrin J et al (2018) Risk of Systemic Adverse Events Associated with Intravitreal Anti-VEGF Therapy for Diabetic Macular Edema in Routine Clinical Practice. Ophthalmology 126(7):1007-1015.

82) Costagliola C, Morescalchi F, Duse S et al (2019) Systemic thromboembolic adverse events in patients treated with intrvitreal anti-VEGF drugs for neovascular age-related macular degeneration: an update. Expert opinion on drug safety vol 18, N 9:803-15.

83) AMD-SID (2018) Standard Italiani per la Cura del Diabete Mellito. http://www.siditalia.it/pdf/Standard\%20di\%20Cura\%20AMD\%20-\%20SID\%202018 protetto2.pdf 
84) Kohner EM and Porta M (1991) European Journal of Ophthalmology 1:45-54.

85) Querques G (2019) Eye complications of diabetes. Acta Diabetologica 56:971. 
Figure legends

Fig. 1 - The VEGF family and receptors. The figure depicts the vascular endothelial growth factor (VEGF) isoforms VEGF (also known as VEGF-A), VEGF-B, VEGF-C, VEGF-D and placental growth factor (P1GF), and their binding to the receptor tyrosine kinases VEGFR-1, VEGFR-2 and VEGFR-3 and the co-receptors neuropilin-1 (NRP-1) and NRP-2. Major effects on receptor-expressing cell types in the vasculature and CNS are indicated. sVEGFR-1 can trap VEGF-A, VEGF-B and PIGF and reduce their biological actions. sVEGFR-1, soluble VEGFR-1.

From: Lange C, Storkebaum E, Ruiz de Almodóvar C, Dewerchin M, Carmeliet P. Vascular endothelial growth factor: a neurovascular target in neurological diseases. Nature Reviews Neurology 12, 439-454, 2016. https://www.nature.com/articles/nrneurol.2016.88/figures/1.

\section{Figure 2. Proliferative diabetic retinopathy}

Top: Image of left eye with new vessels, pre-retinal haemorrhages and diabetic macular edema.

Bottom: Schematic representation of new vessels growing at the vitreo-retinal interface, retinal traction following vitreous detachment and, finally, retinal traction and detachment from newly formed fibrovascular tissue.

Figure 3 - Effects of VEGF blockade.

From: Chen, H. X. \& Cleck, J. N. Nat. Rev. Clin. Oncol. 6, 465-477 (2009)

Fig 4. Structure of anti-VEGF drugs used in the treatment of retinal diseases

From: Thakur S. Health \& Medicine May 10, 2017)

https://www.slideshare.net/drsahilthakur/anti-vegfs-in-ophthalmology 
Table 1. Summary of mayor surveys of cardiovascular events following intraocular administration of anti-VEGF agents.

\begin{tabular}{|c|c|c|c|c|c|c|}
\hline Reference & Type & Eye condition & Agent & N. & Comparator & Cardiovascular outcome \\
\hline PACORES 2008 (58) & Retrospective & DME & Bevacizumab & 1173 & Photocoagulation & Similar to comparator \\
\hline BOLT 2012 (59) & Prospective & CSME & Bevacizumab & 80 & Photocoagulation & Similar to comparator \\
\hline Cheng et al 2012 (72) & Meta-analysis & AMD, DR, RVO & Anti-VEGF & 4942 & No Anti-VEGF & $\begin{array}{l}\text { No increase in arterial } \\
\text { thromboembolic events }\end{array}$ \\
\hline RISE \& RIDE 2013 (61) & Multicentre RCT & DME & $\begin{array}{l}\text { Ranibizumab } \\
0.3 \text { and } 0.5 \mathrm{mg}\end{array}$ & 759 & Sham injections & $\begin{array}{l}\text { Increased risk of adverse events } \\
\text { with } 0.5 \mathrm{mg} \text { Ranibizumab }\end{array}$ \\
\hline CATT/IVAN 2013 (69) & RCT & AMD & Ranibizumab & 738 & Bevacizumab & $\begin{array}{l}\text { More thrombotic events and } \\
\text { heart failure with Ranibizumab }\end{array}$ \\
\hline RESTORE ext 2014 (62) & Multicentre RCT & DME & Ranibizumab & 240 & $\begin{array}{l}\text { Ranibizumab + laser or } \\
\text { laser alone }\end{array}$ & No report on CV events \\
\hline Moya et al 2014 (71) & Cochrane & AMD & Bevacizumab & 3665 & Ranibizumab & $\begin{array}{l}\text { More frequent gastrointestinal } \\
\text { disorders with Bevacizumab }\end{array}$ \\
\hline Thulliez et al 2014 (73) & Meta-analysis & AMD, DME, RVO & $\begin{array}{l}\text { Bevacizumab } \\
\text { Ranibizumab }\end{array}$ & 9557 & No Anti-VEGF & $\begin{array}{l}\text { Increased risk of venous } \\
\text { thromboembolism with } \\
\text { Bevacizumab }\end{array}$ \\
\hline Braithwaite et al 2014 (74) & Cochrane & RVO & Anti-VEGF & 937 & Laser or sham injections & No safety concerns \\
\hline VIVID and VISTA 2016 (63) & Multicentre RCT & DME & Aflibercept & 872 eyes & Laser & Similar to comparator \\
\hline Avery \& Gordon 2016 (75) & $\begin{array}{l}\text { Review and Meta- } \\
\text { analysis }\end{array}$ & DME & $\begin{array}{l}\text { Aflibercept } \\
\text { Ranibizumab }\end{array}$ & 1328 & Laser or sham injections & $\begin{array}{l}\text { Increased risk of death and } \\
\text { cardiovascular incidents }\end{array}$ \\
\hline Hanart et al 2017 (60) & Retrospective & AMD & Bevacizumab & $>5000$ & Photocoagulation & $\begin{array}{l}\text { Increased risk of death from all } \\
\text { causes }\end{array}$ \\
\hline Zarbin et al 2017 (76) & Pooled analysis & DME & Ranibizumab & 1867 & Laser or sham injections & Similar to comparator \\
\hline Thulliez et al 2018 (77) & Overview & AMD, DME, RVO & $\begin{array}{l}\text { Aflibercept } \\
\text { Bevacizumab } \\
\text { Ranibizumab } \\
\end{array}$ & $>30.000$ & No Anti-VEGF & Similar to comparator \\
\hline Virgili et al 2018 (80) & Cochrane & DME & $\begin{array}{l}\text { Aflibercept } \\
\text { Bevacizumab } \\
\text { Ranibizumab }\end{array}$ & 6007 & Each other & $\begin{array}{l}\text { No difference between the } \\
\text { three agents }\end{array}$ \\
\hline Maloney et al 2018 (81) & Retrospective & DME & Anti-VEGF & 23.348 & $\begin{array}{l}\text { Laser or intravitreal } \\
\text { corticosteroid }\end{array}$ & More all-causes hospitalization \\
\hline Starr et al $2019(78)$ & Retrospective & AMD, DME, RVO & Anti-VEGF & 2541 & No Anti-VEGF & Similar to comparator \\
\hline Dalvin et al 2019 (79) & Retrospective & AMD & Anti-VEGF & 504 & No Anti-VEGF & Similar to comparator \\
\hline
\end{tabular}

AMD (Age-related Macular Degeneration), DME (Diabetic Macular Edema), CSME (Clinically Significant Macular Edema), RVO (Retinal Venous Occlusion, RCT (Randomized Controlled Trial),

MRCT (Multicentric Randomized Controlled Trial). 